\title{
Anomaly-Induced Effective Action and Inflation
}

\author{
Julio C. Fabris ${ }^{\text {a }}$, Ana M. Pelinson ${ }^{\mathrm{b}}$ and Ilya L. Shapiro * c \\ a Universidade Federal de Espiríto Santo, Brazil \\ fabris@cce.ufes.br \\ b Centro Brasileiro de Pesquisas Físicas, Rio de Janeiro, Brazil \\ ana@fisica.ufjf.br \\ c Universidade Federal de Juiz de Fora, Brazil \\ Tomsk Pedagogical University, Russia \\ shapiro@fisica.ufjf.br
}

In the early Universe matter can be described as a conformal invariant ultra-relativistic perfect fluid, which does not contribute, on classical level, to the evolution of the isotropic and homogeneous metric. If we suppose that there is some desert in the particle spectrum just below the Planck mass, then the effect of conformal trace anomaly is dominating at the corresponding energies. With some additional constraints on the particle content of the underlying gauge model (which favor extended or supersymmetric versions of the Standard Model rather than the minimal one), one arrives at the stable inflation. We review the model and report about the calculation of the gravitational waves on the background of the anomaly-induced inflation. The result for the perturbation spectrum is close to the one for the conventional inflaton model, and is in agreement with the existing Cobe data.

\section{Introduction}

Inflation is a necessary component of the cosmological standard model. Besides to present a simple solution to the flatness and horizon problems, such issues as metric and density perturbations have been successfully studied in the context of the inflationary model and led to a consistent scenario for structure formation as well as the anisotropies in the relic radiation. But, the inflaton potentials which are necessary to achieve the successful inflation are phenomenological potentials, which can be hardly derived from some quantum field theory. Therefore, there is a lack of a natural model for inflation. An alternative approach to inflation can be based on the trace anomaly and on the effective action of gravity resulting from the quantum effects of matter fields on the classical gravitational background [1 6 .

\footnotetext{
*Authors are grateful to $\mathrm{CNPq}$ (Brazil) for permanent support.
}

\section{Inflationary solution}

Let us describe the general physical input [2 4 ]. Suppose there is a desert in the spectrum of particles which extends to some orders of magnitude below the Planck scale. In the very early Universe the matter may be described by the free radiation, that is, microscopically, by the set of massless fields with negligible interactions between them. Due to the conformal invariance, these fields decouple from the conformal factor of the metric. In this situation the dominating quantum effect is the trace anomaly which comes from the renormalization of the conformal invariant part of the vacuum action (see [7, \&] for the introduction)

$S_{\text {vacuum }}=\int d^{4} x \sqrt{-g}\left\{a_{1} C^{2}+a_{2} E+a_{3} \nabla^{2} R\right\}$

The anomaly-induced effective action can be found explicitly [9, 10,8] with accuracy to an arbitrary conformal functional which vanishes for the special case of the conformally flat metric. The 
expression for the anomaly is:

$$
\begin{aligned}
<T_{\mu}^{\mu}> & =-\frac{2}{\sqrt{-g}} g_{\mu \nu} \frac{\delta \bar{\Gamma}}{\delta g_{\mu \nu}}= \\
& =-\frac{1}{(4 \pi)^{2}}\left(w C^{2}-b E+c \nabla^{2} R\right),
\end{aligned}
$$

where $C^{2}, E$ are the square of the Weyl tensor and the integrand of the Gauss-Bonnet term, and

$w=\frac{N_{0}}{120}+\frac{N_{1 / 2}}{20}+\frac{N_{1}}{10}$

$b=\frac{N_{0}}{360}+\frac{11 N_{1 / 2}}{360}+\frac{31 N_{1}}{180}$

$c=\frac{N_{0}}{180}+\frac{N_{1 / 2}}{30}-\frac{N_{1}}{10}$,

with $N_{0,1 / 2,1}$ - number of fields of spin $(0,1 / 2,1)$. The solution for the effective action can be written in terms of new variables $g_{\mu \nu}=\bar{g}_{\mu \nu} \cdot e^{2 \sigma}$ as:

$$
\begin{aligned}
\bar{\Gamma} & =S_{c}\left[\bar{g}_{\mu \nu}\right]+\frac{1}{(4 \pi)^{2}} \int d^{4} x \sqrt{-\bar{g}}\left\{a \sigma \bar{C}^{2}-\right. \\
& -b \sigma\left(\bar{E}-\frac{2}{3} \bar{\nabla}^{2} \bar{R}\right)-2 b \sigma \bar{\Delta}_{4} \sigma- \\
& \left.\left.-\frac{3 c-2 b}{36}\left[\bar{R}-6(\bar{\nabla} \sigma)^{2}-6\left(\bar{\nabla}^{2} \sigma\right)\right]^{2}\right)\right\},
\end{aligned}
$$

where

$$
\Delta_{4}=\nabla^{4}+2 R^{\mu \nu} \nabla_{\mu} \nabla_{\nu}-\frac{2}{3} R \nabla^{2}+\frac{1}{3}\left(\nabla^{\mu} R\right) \nabla_{\mu}
$$

is the fourth derivative, conformal invariant and self-adjoint operator and $S_{c}\left[\bar{g}_{\mu \nu}\right]$ is some unknown functional of the metric $\bar{g}_{\mu \nu}(x)$ which serves as an integration constant for the effective action. This action includes some arbitrariness, which was extensively investigated recently. It is accepted that all the arbitrariness is inside the conformal functional $S_{c}\left[\bar{g}_{\mu \nu}\right]$.

The induced action can be presented in a nonlocal but covariant form using the original metric and then in a local covariant form via auxiliary scalars [11]. The most useful local form of the action is:

$$
\begin{aligned}
\bar{\Gamma} & =S_{c}\left[g_{\mu \nu}\right]-\frac{3 c-2 b}{36(4 \pi)^{2}} \int d^{4} x \sqrt{-g(x)} R^{2}+ \\
& +\int d^{4} x \sqrt{-g(x)}\left\{\frac{1}{2} \varphi \Delta_{4} \varphi-\frac{1}{2} \psi \Delta_{4} \psi\right.
\end{aligned}
$$

$$
\begin{aligned}
& +\varphi\left[\frac{\sqrt{b}}{8 \pi}\left(E-\frac{2}{3} \nabla^{2} R\right)-\frac{w}{8 \pi \sqrt{b}} C^{2}\right] \\
& \left.+\frac{w}{8 \pi \sqrt{b}} \psi C^{2}\right\},
\end{aligned}
$$

where $\varphi, \psi$ are auxiliary fields [9, 11,5].

Consider the inflationary solution for the theory

$S_{\text {total }}=-M_{P}^{2} \int d^{4} x \sqrt{-g} R+\bar{\Gamma}$,

where $M_{P}^{2}=1 / 16 \pi G$ is the square of the Planck mass, and the quantum correction $\bar{\Gamma}$ is taken from the anomaly-induced action. Since such a strategy was justified in the black hole case [11], one can set $S_{c}\left[g_{\mu \nu}\right]=0$, including to it, when it is not indicated explicitly, also the classical vacuum term.

We look for the isotropic and homogeneous solution $g_{\mu \nu}=a^{2}(\eta) \bar{g}_{\mu \nu}$, where $\eta$ is conformal time. It proves useful to denote $\sigma=\ln a$. We shall consider the conformally flat background and thus set $\bar{g}_{\mu \nu}=\eta_{\mu \nu}$.

The equations for the auxiliary fields:

$$
\begin{aligned}
& \Delta_{4} \varphi+\frac{\sqrt{b}}{8 \pi}\left(E-\frac{2}{3} \nabla^{2} R\right)-\frac{w}{8 \pi \sqrt{b}} C^{2}=0 \\
& \Delta_{4} \psi-\frac{w}{8 \pi \sqrt{b}} C^{2}=0 .
\end{aligned}
$$

reduce to

$\nabla^{4} \varphi+\frac{\sqrt{b}}{2 \pi} \nabla^{4} \sigma=0, \quad \nabla^{4} \psi=0$.

The solutions

$\varphi=-\frac{\sqrt{b}}{2 \pi} \sigma+\varphi_{0}, \quad \psi=\psi_{0}$.

where $\varphi_{0}, \psi_{0}$ are general solutions of the homogeneous equations

$\nabla^{4} \varphi_{0}=0, \quad \nabla^{4} \psi_{0}=0$.

Thus one meets an arbitrariness related to the choice of the initial conditions for the auxiliary fields. Indeed, the inflationary solution does not depend nor on $\varphi_{0}, \psi_{0}$ neither on $S_{c}\left[g_{\mu \nu}\right]$. The difference shows up only when we investigate the metric perturbations. 
In terms of the physical time $t$, defined, as usual, through $a(\eta) d \eta=d t$ and $H(t)=$ $\dot{a}(t) / a(t)=\dot{\sigma}(t)$, the equation is

$$
\begin{gathered}
\dddot{H}+7 \ddot{H} H+4\left(1+\frac{3 b}{c}\right) \dot{H} H^{2}+4 \dot{H}^{2} \\
+\frac{4 b}{c} H^{4}-\frac{2 M_{P}^{2}}{c}\left(H^{2}+\dot{H}\right)=0 .
\end{gathered}
$$

The special solution corresponding to constant $H$ :

$H= \pm \frac{M_{P}}{\sqrt{b}}, \quad a(t)=a_{0} \cdot \exp H t$.

Positive sign corresponds to inflation.

This solution was discovered and investigated by Starobinsky and others in 80'th [2.3. 3]. Also, there are two other similar solutions for the FRW metric with $k= \pm 1$. The special inflationary solution is stable with respect to the variations (not necessary small) of the initial data for $a(t)$, if the parameters of the underlying quantum theory satisfy the condition $\frac{b}{c}>0$, that leads to

$N_{1}<\frac{1}{3} N_{1 / 2}+\frac{1}{18} N_{0}$.

This constraint is not satisfied for the MSM with $N_{1}=12, N_{1 / 2}=24$ and $N_{0}=4$.

However, one can consider some aspects of the neutrino oscillations as an indication that the MSM should be extended. Below we consider two versions, each of them leads to stable inflation.

i) Extended SM: $N_{1}=12, N_{1 / 2}=48, N_{0}=8$ and

ii) MSSM $N_{1}=12, N_{1 / 2}=32, N_{0}=104$.

The advantage of stable inflation is that it occurs independent of the initial data. After the Big Bang, when the Universe started to expand and the typical energy decreased below the Planck order, we can imagine some kind of "string phase transition". Starting from this point, the effective quantum field theory is an adequate description, and the anomaly-induced model applies. In case of the stable inflation, the initial data for $a(t)$ and its derivatives do not need to be fine tuned, if only the condition $18 N_{1}<6 N_{1 / 2}+N_{0}$ is satisfied - the inflation is unavoidable.

Let us calculate the necessary duration of inflation. Suppose we want the Universe to expand in $n$ e-folds. Then the total rate of inflation, in the Planck units, is

$\frac{a\left(t_{0}+\delta t\right)}{a\left(t_{0}\right)}=\exp \left\{4 \pi \sqrt{\frac{360}{N_{t}}} \delta t\right\}$

where $N_{t}=N_{0}+11 \cdot N_{1 / 2}+62 \cdot N_{1}$, and thus

$\delta t=\frac{1}{4 \pi} \sqrt{\frac{N_{t}}{360}} \cdot n$.

The time necessary for 65 e-folds is around ten Planck times only.

The numerical study has shown that the exponential solution stabilizes in much shorter time. One can safely derive the metric perturbations on the exponentially inflating background, independent on the initial data.

The most difficult question is how the inflation ends. So far, we do not have a definite answer to this question, but there are some particular indications that a solution is possible if we take the masses of the matter particles (perfect fluid) into account [1]. A very important observation is that even when the Universe expands so rapidly, the average energy of the particles decreases. At some instant it decreases such that their masses become relevant and then the matter part of the equation gets some dust component. In this case inflation is not anymore a solution. The classical solution for dust $a(t) \sim t^{2 / 3}$ also is not a solution because of the quantum term. However, in this case both Einstein and matter terms behave like $t^{-2}$ while the "quantum" part behaves like $t^{-4}$, and very rapidly the anomaly-induced quantum term becomes irrelevant. Thus, one can suppose that at the long-time limit $a(t) \sim t^{2 / 3}$ is a good approximation for the unknown solution of the equation with matter. Of course, the above consideration is not a solution of the problem, but one can hope that the solution will be found along this line.

Let us now review the equation for the metric perturbations which is based on the bilinear expansion of the action $\llbracket$.

Now we have to fix the arbitrariness related to the homogeneous solutions $\varphi_{0}$ and $\psi_{0}$ in the inflationary solution. The choice of initial data for $\varphi$ and $\psi$ defines the vacuum state for the perturbations. But, what could be the criterion for the choice? 
One can make a very useful comparison with the vacuum of the black hole background [11. In the black hole case (semi-classical approach) the vacuum which provides a smooth transition to the Minkowski vacuum at the space infinity is the Boulware one. Let us suppose that the proper cosmological vacuum for the expanding Universe reduces to the Minkowski one at infinite time. Then the detailed consideration [5] gives

$\varphi=-\frac{\sqrt{b}}{2 \pi} \sigma, \quad \psi=0$.

With this choice, one can perform the numerical study of the equation for the perturbations. The technical details can be found in [5].

A numerical analysis requires that the equations must be dimensionless and the initial conditions must be consistently chosen. For the first point, if one sets the Planck mass equal to one, time is automatically measured in the Planck units. For the initial conditions, we consider that the perturbations have a quantum origin: the seed of the perturbations are quantum fluctuations of the primordial fields [5].

Using the dimensionless equation and taking the initial conditions consistent with an initial quantum spectrum, one can integrate the fourth order equation numerically. A crucial number is the power spectrum of the perturbations, which in the long wavelength limit (it is the most important for cosmology) is of the type $P_{n}^{2} \propto n^{k}$. This distribution tells how the amplitude of the perturbations depends on $n$. The coefficient $k$ is called spectral indice of the perturbation.

In the standard DeSitter case [12] one meets exact result $k=0$. The Cobe, Boomerang and Maxima observational programs favor the flat spectrum too, with the bounds $-0.15<k<0.16$ (see, for example, 13]). For our model, the numerical procedure gives [5] $k \simeq-0.01$ which is qualitatively in agreement with a flat spectrum.

Hence, our model predicts a spectral indice different, but not very far from the one predicted by the traditional inflationary scenario. Besides, the prediction has quite a good agreement with the observational results.

As a conclusion, we consider the stable anomaly-induced model [4.5] as the most natural one, because it does not require any fine-tuning, nor for inflation neither for the spectrum of metric perturbations. The only serious problem is the grace-exit, which can be hopefully solved in the framework of effective approach.

\section{REFERENCES}

1. M.V. Fischetti, J.B. Hartle and B.L. Hu, Phys.Rev. D20 (1979) 1757.

2. A.A. Starobinski, Phys.Lett. 91B (1980) 99; JETP Lett. 34 (1981) 460.

3. A. Vilenkin, Phys.Rev. D32 (1985) 2511.

4. J.C. Fabris, A.M. Pelinson, I.L. Shapiro, Grav. Cosm. 6 (2000) 59. gr-qc/9810032]; Vacuum effective action and inflation. hepth/9912040. (Proceedings of A. Swieca School, 1999. To be published.)

5. J.C. Fabris, A.M. Pelinson, I.L. Shapiro, On the gravitational waves on the background of anomaly-induced inflation. hep-th/0009197].

6. S.W. Hawking, T. Hertog and H.S. Real, Trace anomaly driven inflation. hepth/0010232.

7. N.D. Birell and P.C.W. Davies, Quantum fields in curved space (Cambridge Univ. Press, Cambridge, 1982).

8. I.L. Buchbinder, S.D. Odintsov and I.L. Sh., Effective Action in Quantum Gravity. (IOP, 1992).

9. R.J. Reigert, Phys.Lett. 134B (1980) 56.

10. E.S. Fradkin and A.A. Tseytlin, PhLB134(1980)187.

11. I. Shapiro and A. Jacksenaev, Phys.Lett. 324B (1994) 284;

R. Balbinot, A. Fabbri and I. Shapiro, Phys.Rev.Lett. 83 (1999) 1494; Nucl.Phys. B559 (1999) 301.

12. L.P. Grishchuk, Phys.Rev. D48 (1993) 3513.

13. W. Hu, M. Fukugita, M. Zaldarriaga and M. Tegmark, astro-ph/0006436. 